\title{
Command-destruct thermosets via photoinduced thiol-catalyzed ß-scission of acyclic benzylidene acetals +
}

\author{
William D. Walker, ${ }^{a}$ Michael J. Sandoz, ${ }^{a}$ Samuel Roland, ${ }^{a}$ Tony M. Buster, ${ }^{a}$ John K. Newman, ${ }^{b}$ and Derek L. \\ Patton*a \\ ${ }^{a}$ School of Polymer Science and Engineering, University of Southern Mississippi, Hattiesburg, MS 39406. E-mail: \\ derek.patton@usm.edu

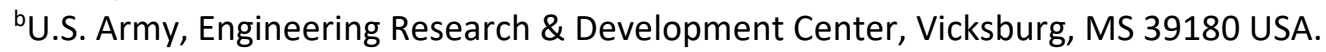

\begin{abstract}
Photoinduced thiol-catalyzed hydrogen abstraction and ßscission of acyclic benzylidene acetals is demonstrated as a new route to "command-destruct" polymer thermosets. Using this approach, we show that poly(thioether acetal) networks synthesized via thiol-ene photopolymerization rapidly degrade to alkyl benzoate byproducts when triggered with light, transitioning from solid to liquid within seconds. The lightdriven construction and destruction processes, accessible via distinct differences in kinetics, are readily amendable for photopatterning, additive/subtractive manufacturing and wavelength-selective applications.
\end{abstract}

Sulfur-centered radicals are ubiquitous in many chemical and biological processes ${ }^{1}$ and have found broad utility in synthetic polymer chemistry due to their facile formation and inherent reactivity. ${ }^{2,3}$ Due to the relatively weak $\mathrm{S}-\mathrm{H}$ bond (bond dissociation energy $79-87 \mathrm{kcal} / \mathrm{mol}),{ }^{4}$ thiyl radicals are readily generated by hydrogen atom abstraction of thiols via thermal or photochemical initiation. Once formed, thiyl radicals undergo efficient addition to a broad range of unsaturated motifs such as alkenes and alkynes. Collectively, these attributes have served as the foundation for the development of thiol-ene $e^{5,6}$ and thiol$y n e^{7,8}$ reactions as versatile methodologies for the synthesis and modification of functional polymeric materials.

While charge neutral, radicals tend to exhibit characteristics of either electrophilic or nucleophilic reactive intermediates. Thiyl radicals typically behave as electrophilic intermediates readily abstracting hydrogen atoms from weak, electron-rich $\mathrm{C}$ $\mathrm{H}$ bonds - a process that is particularly favored for $\mathrm{C}-\mathrm{H}$ bonds linked to heteroatoms (e.g. aliphatic alcohols/amines, acetals, etc.). ${ }^{1} \mathrm{H}$-abstraction then generates a nucleophilic carboncentered radical that can either be reduced back the starting material by the thiol or engage in additional transformations. Roberts et al. exploited the concept of converting electrophilic radicals to nucleophilic radicals using catalytic quantities of thiol as "polarity reversal catalysts" (PRCs) for a broad range of small molecule transformations, ${ }^{9}$ including reductions, ${ }^{10}, 11$ epimerizations, ${ }^{12}$ and deoxygenations. ${ }^{13}$ Uchiyama et al. recently employed thiols as PRCs for the reduction of RAFT polymer chain ends; ${ }^{14}$ however, despite the ubiquity of thiols in polymer chemistry, the PRC concept remains underutilized.

Building on our recent work with hydrolytically degradable poly(thioether acetal) thermosets, ${ }^{15-17}$ we were inspired by literature from Roberts et al. that used thiols as PRCs for radical- mediated redox of benzylidene acetals to benzoate esters (Fig. 1). ${ }^{18-21}$ In these reactions, electrophilic thiyl radicals facilitate $\mathrm{H}$ atom abstraction at the electron-rich acetal $\mathrm{C}-\mathrm{H}$ bond, resulting in a carbon-centered dialkoxybenzyl radical that undergoes $B$ scission to give a benzoate ester and a nucleophilic alkyl radical - the latter of which readily abstracts a hydrogen from a thiol to form the product and a thiyl radical continuing the chain reaction. For cyclic benzylidene acetals, the overall reaction represents a redox rearrangement where the benzoate ester and alkyl functional groups remain joined (Fig. 1A). However, application of the thiol-catalyzed process to acyclic benzylidene acetals, such as those employed as building blocks for our previous work, ${ }^{15-17}$ would result in cleavage of the acetal to the benzoate ester and alkyl group ${ }^{22}$ (Fig. 1B) - a process that we postulated could lead to polymer network destruction.

Herein, we report the design of command-destruct thermosets based on photoinduced thiol-catalyzed ß-scission of acyclic benzylidene acetals. The process enables rapid thiol-ene photopolymerization and subsequent photodegradation of poly(thioether acetal) thermosets using the same light source with simple temporal control - a salient consequence of the distinct difference in kinetics of the radical-mediated photopolymerization and thiol-catalyzed radical ß-scission processes. We demonstrate how molecular level ß-scission reactions within a step-growth network can be translated into rapid macroscopic transformations of the physical state of the thermoset (e.g. solid to liquid) upon exposure to light. Using stoichiometrically imbalanced thiol-ene formulations, we show that free thiols within the networks are critical for the destruction process. Since the degradation reaction is driven by

Thiols as "polarity reversal catalysts" for acetal redox reactions

(A) Previous work: Cyclic benzylidine acetals (rearrangement)

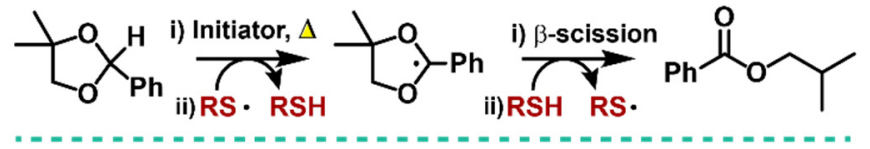

(B) This work: Acyclic benzylidine acetals (cleavage)

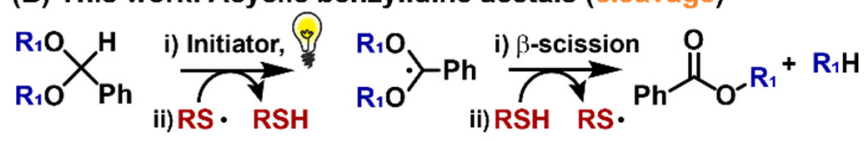

Fig. 1 (A) Previous work demonstrating radical-mediated redox rearrangement of cyclic benzylidene acetals to alkyl benzoate esters using thiols as a polarity reversal catalyst. (B) Proposed method leveraging thiols as polarity reversal catalysts for cleavage of acyclic benzylidene acetals. 
(A) Thiol-Ene

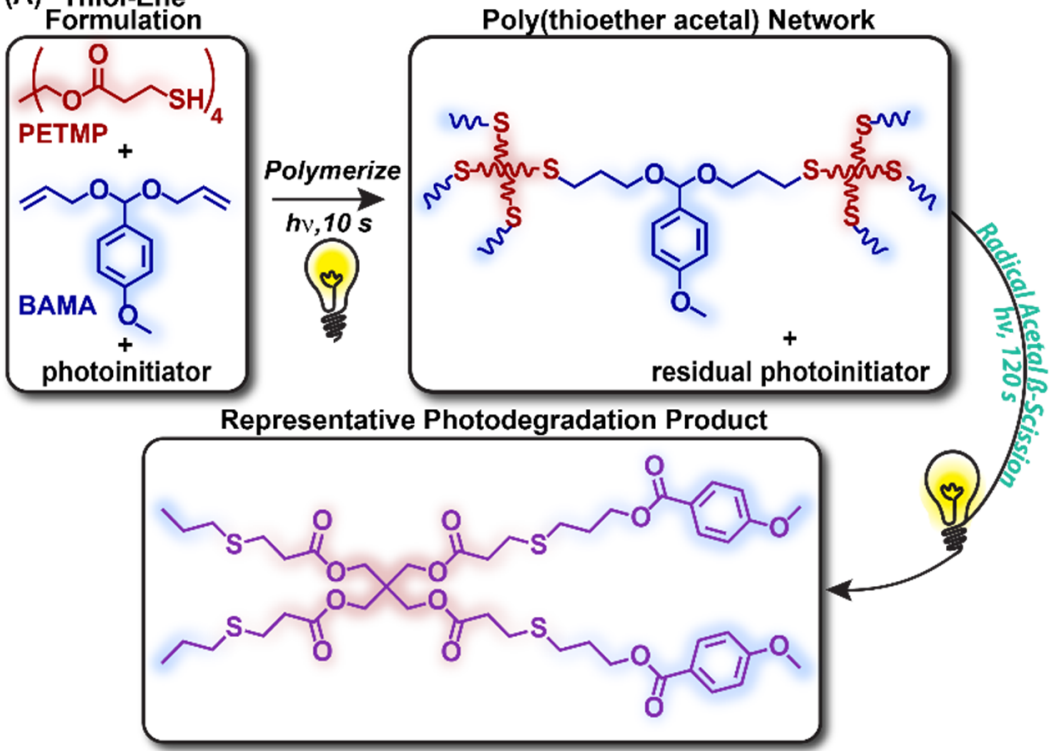

(B)

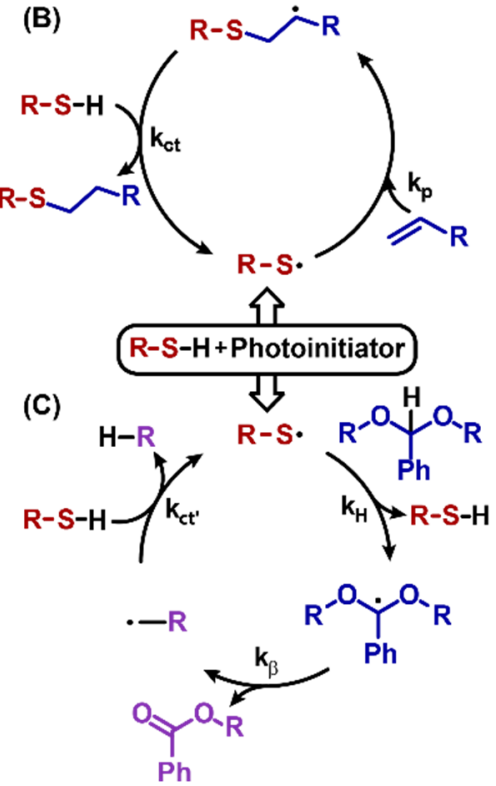

Fig. 2 (A) Synthesis of poly(thioether acetal) photopolymer network from PETMP and BAMA monomers and subsequent degradation to alkyl benzoate species, (B) Mechanism of radical-mediated step-growth thiol-ene photopolymerization, and C) mechanism of thiol-catalyzed B-scission of acetals to alkyl benzoates.

light, the platform is readily amendable for spatial control (demonstrated via simple photolithography) and wavelength selectivity (demonstrated via judicious choice of photoinitiator and light source).

Fig. 2A shows the synthetic route for command-destruct poly(thioether acetal) thermosets via thiol-ene photopolymerization. Bis-allyl p-methoxybenzylidene acetal (BAMA) and pentaerythritol tetrakis(3-mercaptopropionate) (PETMP) were selected as a model building blocks based on our previous work. ${ }^{15-17}$ The reaction cycles for thiol-ene photopolymerization and thiol-catalyzed photodegradation of acyclic benzylidene acetals are illustrated in Fig. 2B and 2C, respectively. Thiol-ene photopolymerization proceeds via a radical-mediated step-growth mechanism. The two-step process involves addition of a thiyl radical across a double bond to form a carbon-centered radical intermediate, which then undergoes chain transfer with another thiol to generate the thioether adduct and regenerate the propagating thiyl radical. Acyclic benzylidene acetals are postulated to undergo cleavage to give benzoate esters via the formation and subsequent $\beta$-scission of a dialkoxybenzyl radical with thiols serving to catalyze the hydrogen abstraction and transfer processes. Since thiols and thiyl radicals are common to both photopolymerization and photodegradation processes, successful implementation of this photodegradation process requires 1 ) the availability of free thiols after polymerization and 2) the photopolymerization kinetics to be faster than the photodegradation kinetics. These requirements, and elucidation of the photodegradation mechanism, are further explored below.

The requirement of free thiols within the polymer network to catalyze the photodegradation process was explored by exploiting the step-growth thiol-ene mechanism and stoichiometric imbalance in the monomer formulation. BAMA and PETMP were combined in 1:1, 1.1:1, and 1:1.1 thiol:alkene mole ratios to fabricate samples with stoichiometric thiol and alkene functionality, excess thiol functionality, and excess alkene functionality, respectively. These formulations, with Darocur 1173 (10 mol\% relative to the acetal crosslinks) as a photoinitiator, were placed between two glass slides separated by $500 \mu \mathrm{m}$ PTFE spacers and cured with UV light $(200 \mathrm{~mW} / \mathrm{cm}$, $\lambda_{\max }=365 \mathrm{~nm}$ ) for $3 \mathrm{~s}$ per side (kinetics are discussed below). Samples were kept dry to avoid hydrolytic degradation of the acetal linkages. ${ }^{16,17}$ After photopolymerization, the samples were removed from the glass molds and subsequently exposed to UV light ( $400 \mathrm{~mW} \mathrm{~cm}^{-1}, \lambda_{\max }=365 \mathrm{~nm}$ ) as a function of time to investigate the photodegradation behavior. As shown in Fig. 3A, the thermoset containing $10 \mathrm{~mol} \%$ excess thiol underwent rapid photoinduced degradation illustrated clearly by the change in physical state from solid to viscous liquid in less than $60 \mathrm{~s}$ (see Video S1). In contrast, thermosets fabricated with stoichiometric thiol:ene ratios exhibited minor changes in appearance but remain mostly tack-free (Fig. 3B), while systems with $10 \mathrm{~mol} \%$ excess alkene appeared to be completely stable, showing minimal changes over the same timescale of UV exposure (Fig. 3C).

To investigate the photodegradation process in more detail, the liquid byproducts from degradation were collected for analysis. Removal of low molecular weight photoinitiator fragments under reduced pressure yielded a viscous yellow oil. The ${ }^{1} \mathrm{H}$ NMR spectrum of the crude mixture is shown in Fig. S1. The lack of an appreciable aldehyde peak between 9.89-10.1 ppm indicates that neither hydrolysis nor photoinduced acetal decaging ${ }^{23}$ is responsible for the observed degradation process. The ${ }^{1} \mathrm{H}$ NMR spectrum and proposed structure of the major degradation product isolated via flash chromatography are shown in Fig. 3D (blue spectrum). Aromatic peak assignments ( $\delta$ $=7.99 \mathrm{ppm}, \mathrm{J}=8.90 \mathrm{~Hz} ; \delta=6.91 \mathrm{ppm}, \mathrm{J}=8.90 \mathrm{~Hz} ; \Delta_{\delta}=1.08 \mathrm{ppm}$ ), shifted downfield from the acetal monomer and $p$ - 
(A)

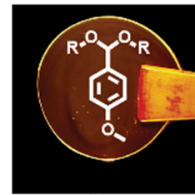

(B)

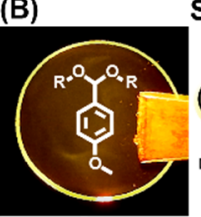

$0 \mathbf{s}$

(C)

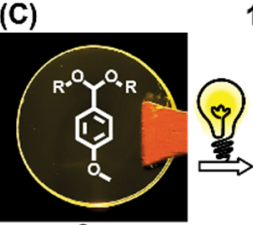

$0 \mathrm{~s}$
$10 \%$ Excess Thiol

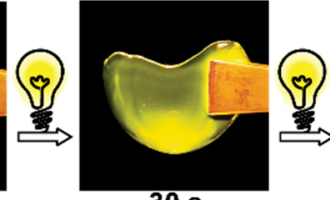

$30 \mathrm{~s}$

Stoichiometric Thiol:Ene
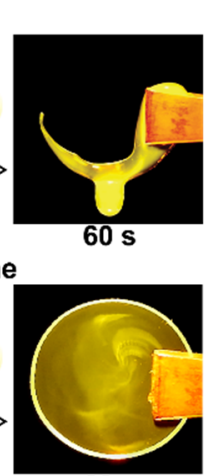

$60 \mathrm{~s}$

'(D)

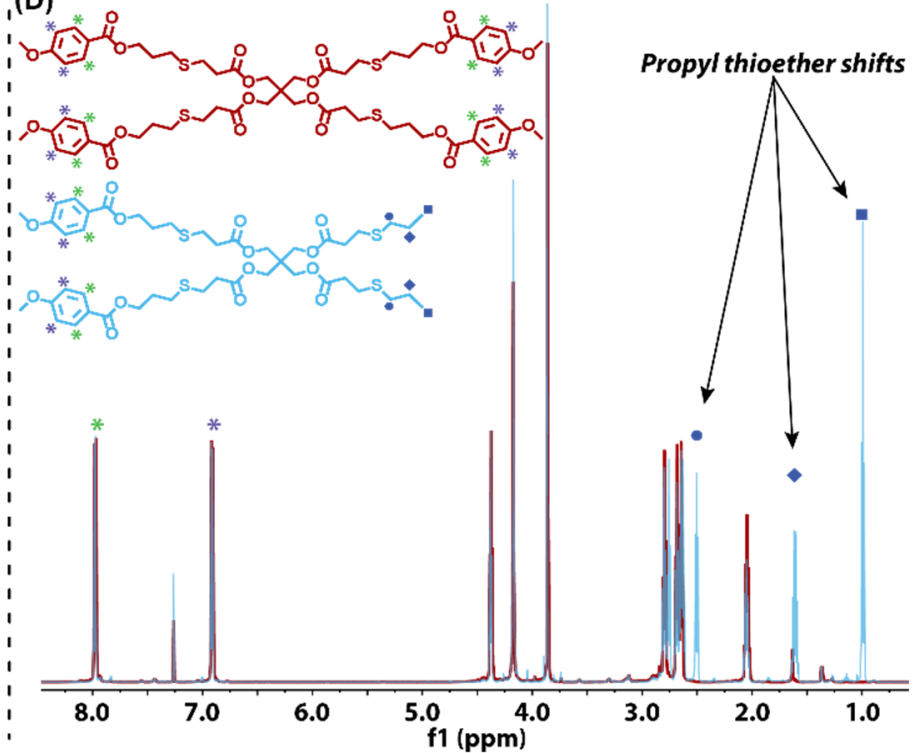

Fig. 3 Snapshots as a function of UV light exposure time for poly(thioether acetal) disks containing (A) 10 mol\% excess thiol, (B) stoichiometric thiol to

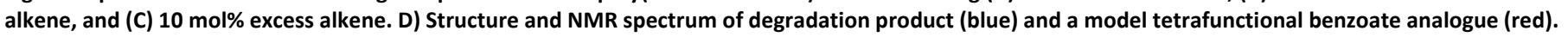

methoxybenzaldehyde, are consistent with the presence of a benzoate ester functional group and are in good agreement with ${ }^{1} \mathrm{H}$ NMR assignments for the tetrafunctional $p$-methoxybenzoate model analog (prepared from PETMP and allyl 4methoxybenzoate for comparison) shown in Fig. 3D (red spectrum). The major degradation product also exhibited peaks at $2.49,1.59$, and $0.97 \mathrm{ppm}$ which are assigned to the terminal propyl thioether chains present in the proposed structure. Structural confirmation of the degradation byproduct is consistent with the thiol-catalyzed hydrogen abstraction and radical ß-scission mechanism.

The kinetics of the thiol-ene polymerization and thiolcatalyzed ß-scission processes were simultaneously measured via real-time transmission FT-IR spectroscopy under a UV light source $\left(200 \mathrm{~mW} \mathrm{~cm}^{-1}, \lambda_{\max }=365 \mathrm{~nm}\right)$. The polymerization kinetics were characterized following the change in thiol peak at $2550 \mathrm{~cm}^{-1}$ over time. After establishing a baseline, the light "on" timepoint is indicated by the vertical dashed line. Representative kinetic profiles for resins formulated with 1:1, 1.1:1, and 1:1.1 thiol:alkene mole ratios are shown in Fig. 4A. Polymerizations showed rapid ( $<5 \mathrm{~s}$ of UV exposure), quantitative thiol conversions for samples formulated with 1:1 and 1:1.1 thiol:ene stoichiometries, whereas thiol conversion expectedly plateaued at approximately $90 \%$ for samples formulated with 1.1:1 thiol:ene stoichiometry. The photodegradation kinetics were characterized by tracking the appearance of the benzoate ester peak $\left(774 \mathrm{~cm}^{-1}\right)$, which according to the mechanism in Fig. $2 \mathrm{C}$ is indicative of $ß$-scission of the acetal linkage. Fig. 4B shows the photodegradation kinetic profiles for samples formulated with various thiol:ene stoichiometries and 10 mol\% photoinitiator. As shown, the thiol:ene stoichiometry significantly influences of the extent acetal ß-scission within the polymer network. Samples with 10 mol\% excess thiol exhibit a rapid increase in benzoate ester upon exposure to UV light. However, in the absence of excess thiols to catalyze the reaction, the appearance of the benzoate ester is significantly diminished as indicated in the kinetic profiles for samples with equal thiol:ene stoichiometry or 10 mol\% excess alkene (Fig. 4B). Here, it is important to note the stark difference

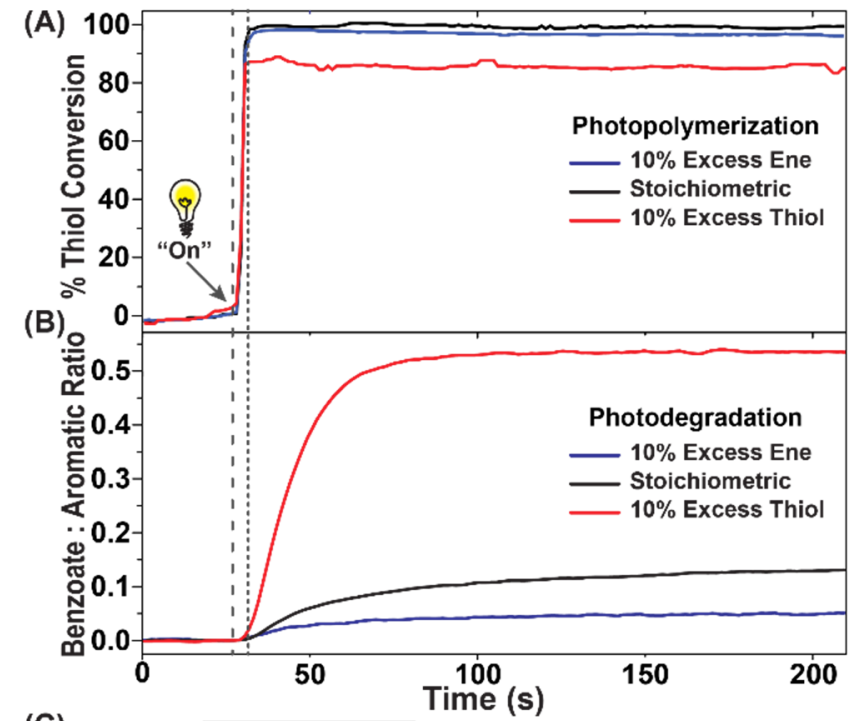

(C)

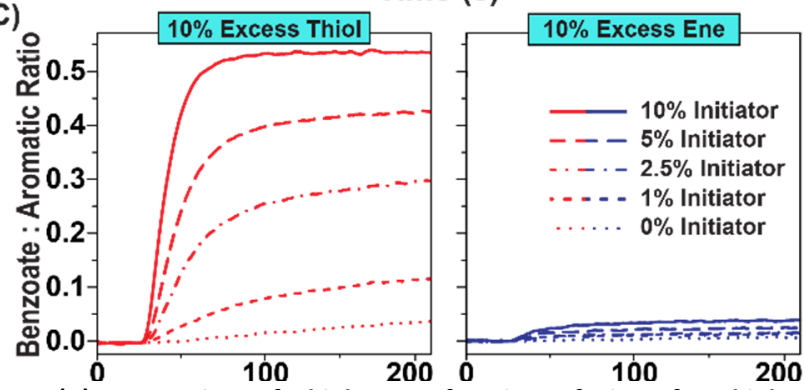

Fig. 4. (A) Conversion of thiol as a function of time for thiol-ene photopolymerization conducted with various thiol:ene stoichiometries, (B) Formation of benzoate as a function of UV exposure time for (B) samples prepared from various thiol:ene stoichiometries, and samples with $10 \mathrm{~mol} \%$ excess thiol (C) and alkene (D) containing various photoinitiator concentrations 
in apparent rates of the photopolymerization and photodegradation processes by comparing the kinetic profiles in Fig. 4A and 4B. Clearly, the kinetics for thiol-ene photopolymerization that form the polymer network are appreciably faster than the kinetics of degradation. Photopolymerization achieves $>85 \%$ thiol conversion prior to significant onset of the $ß$-scission process ( $<5 \%$ benzoate ester at the same time point of light exposure), as indicated by the vertical dotted lines in Fig. 4A and 4B. While a detailed kinetics analysis is beyond the scope of this communication, the observed trends are consistent with reported magnitudes for propagation and chain-transfer kinetic constants for thiol-allyl ether polymerization $\left(10^{5}-10^{6} \mathrm{M}^{-1} \mathrm{~s}^{-1}\right)^{2}, 24,25$ and kinetic constants for acyclic benzylidene acetal $\mathrm{C}-\mathrm{H}$ hydrogen abstraction $\left(10^{5} \mathrm{M}^{-1} \mathrm{~s}^{-1}\right) .{ }^{22}$ Insufficient data is currently available to describe the kinetic constants for the ß-scission of benzylidene acetals. ${ }^{22}$ Nonetheless, these initial kinetic profiles highlight the ability to carry out photopolymerization and photodegradation processes independently using the same light source with temporal control. Judicious design of the acetal monomer (e.g. choice of alkene, benzylidene substituents, etc.) is underway to further distinguish these construct and destruct processes. Finally, the role residual photoinitiator plays in the degradation process warrants discussion. As expected, residual photoinitiator is necessary to generate thiyl radicals to facilitate the abstraction and B-scission processes for photoinduced degradation. As shown in Fig. 4C, an increase in photoinitiator loading from $0-10 \mathrm{~mol} \%$ results in an increased rate of photodegradation in samples containing 10 mol\% excess thiol. Radicals generated by photolysis of the residual initiator may also abstract the acetal hydrogen resulting in ß-scission; however, this process is inefficient in the absence of the thiol to serve as a polarity reversal catalyst. In the absence of thiol, continuation of the chain reaction cycle requires direct hydrogen atom transfer between two nucleophilic carbon-centered radicals (e.g., alkyl and benzylidene radicals) - a process disfavored based on adverse polarity effects. ${ }^{9}$ Evidence to this point is clearly shown in kinetic profiles for samples containing a stoichiometric excess of alkene as a function of photoinitiator loading (Fig. 4D). Even at 10 mol\% photoinitiator, the conversion from acetal to benzoate ester remains relatively low in the absence of thiol.

An attractive feature of using light as a stimulus for degradation is the ability to apply it in a local or spatially resolved fashion using either a well-defined light source or a simple photomask. The light-mediated thiol-ene and ß-scission processes described in the current work are well-suited for photolithographic approaches, enabling both construction and destruction of the polymer resin using the same light source. This concept was explored using the simple photolithography workflow shown in Figure 5A. A poly(thioether acetal) thermoset disk $(0.5 \mathrm{~mm}$ thick $\times 40 \mathrm{~mm}$ diameter, 10 mol\% excess thiol, 10 mol\% Darocur 1173) was first fabricated via exposure to UV light for $3 \mathrm{~s}$. Once cured, the disk was covered with a photomask and irradiated with UV light $\left(600 \mathrm{~mW} \mathrm{~cm}^{-2}, \lambda_{\max } 365 \mathrm{~nm}\right)$ for $120 \mathrm{~s}$. The photomask was then removed, and the disk was washed
(A)
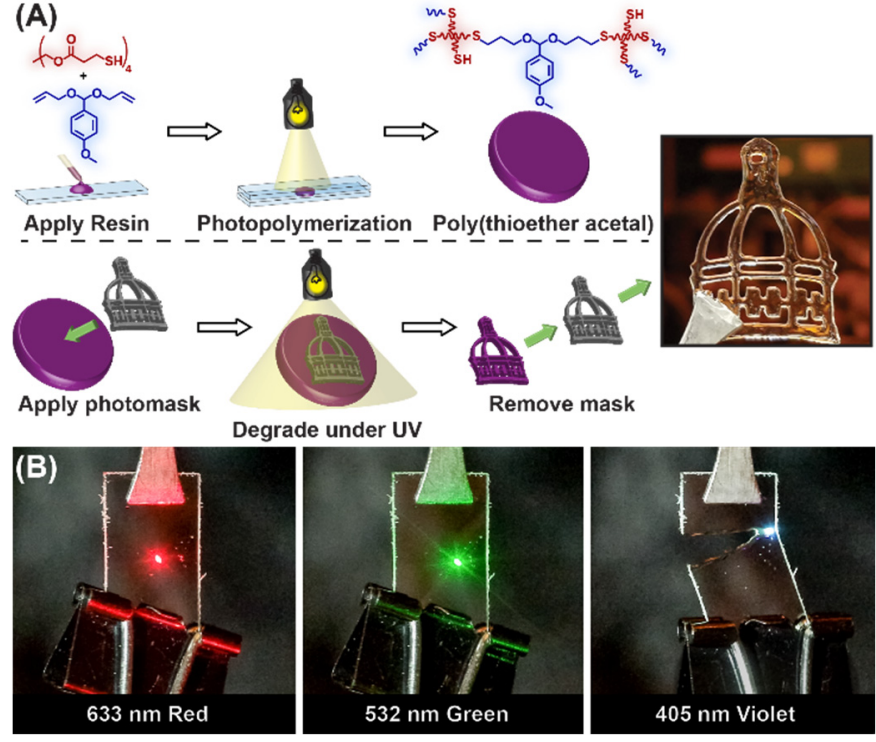

Fig. 5 (A) Simple demonstration of poly(thioether acetal) thermoset construction containing excess thiols and spatially resolved degradation via shadow mask photolithography. (B) Wavelength selective photodegradation of the poly(thioether acetal) thermoset using a $405 \mathrm{~nm}$ laser to interact with the TPO-L photoinitiator; red and green lasers have no spectral overlap with TPO-L.

with hexanes to remove the liquid degradation byproducts within the exposed regions yielding the patterned structure with good edge fidelity. In the classic sense, the benzylidene acetalbased resin functions as positive photoresist in this simple demonstration; however, these resins should be readily amenable to 3D printing and erasing processes (e.g. additive and subtractive manufacturing) following the examples of similar reports based on photocleavable 0 -nitrobenzyl groups. ${ }^{26,27}$ An additional element that is easily exploited in the current resin platform is wavelength selectivity for the photodegradation process via choice of photoinitiator. Here, poly $(\beta$-thioether acetal) films were fabricated (10 mol\% excess thiol, $10 \mathrm{~mol} \%$ Omnirad TPO-L) via exposure to UV light for $3 \mathrm{~s}$. The Omnirad TPO-L photoinitiator was selected for its absorbance overlap with the near UV region of the spectrum, with the $\lambda_{\max }$ at $383 \mathrm{~nm}$ extending out past $405 \mathrm{~nm} .{ }^{28}$ Wavelength selective degradation of these films was demonstrated using three different lasers, including $50 \mathrm{~mW} 405 \mathrm{~nm}$ (violet), $100 \mathrm{~mW} 532 \mathrm{~nm}$ (green), and $150 \mathrm{~mW} 633 \mathrm{~nm}$ (red). As shown in Figure 5B (and Video S2), irradiation of the films with the green and red lasers caused no photochemical response due to the lack of spectral overlap of TPO- $L$ with the green and red wavelengths. However, the films underwent rapid photodegradation via thiol-catalyzed radical $ß$ scission of benzylidene acetals following the beam path of the violet laser, as it was the only laser source in the series to interact with the TPO-L photoinitiator.

\section{Conclusions}

In summary, we demonstrated a new and facile approach to synthesize command-destruct poly(thioether acetal) thermosets via thiol-ene photopolymerization and photoinduced thiol-catalyzed radical ß-scission of acyclic 
benzylidene acetals. These materials undergo rapid photoinduced degradation, where thiols serve as polarity reversal catalysts to facilitate scission of benzylidene acetal network junctions. The stark difference in kinetics of the photopolymerization and photodegradation processes enables the construction and destruction of the polymer network using a common light source under temporal and/or spatiotemporal control. Spatially controlled degradation of the thermosets was achieved using simple photolithography techniques to create patterned structures, whereas wavelength selective degradation was demonstrated using a photoinitiator sensitive to violet laser light. This concept - highlighting photoinduced command-construct and command-destruct processes exhibits potential for a range of applications, including degradable adhesives, additive/subtractive manufacturing, and more broadly as environment friendly materials with welldefined end-of-use management strategies.

\section{Conflicts of interest}

There are no conflicts to declare.

\section{Acknowledgements}

The authors acknowledge the National Science Foundation (CHE-1710589) and the U.S. Army Engineer Research and Development Center (Contract No. W912HZ-18-C-0022) for partial support of this research. WDW acknowledges traineeship support from the NSF NRT program "Interface" (DGE-1449999) through the University of Southern Mississippi.

\section{Supporting Information}

+Supporting information available: Experimental/synthetic details, FTIR, NMR, degradation videos.

\section{Notes and references}

1. F. Dénès, M. Pichowicz, G. Povie and P. Renaud, Chem. Rev., 2014, 114, 2587-2693.

2. C. E. Hoyle and C. N. Bowman, Angew. Chem. Int. Ed., 2010, 49, 1540-1573.

3. M. J. Kade, D. J. Burke and C. J. Hawker, J. Polym. Sci. A Polym. Chem., 2010, 48, 743-750.

4. Y. Luo, Handbook of Bond Dissociation Energies in Organic Compounds, CRC Press, Boca Raton, FL, 2003.

5. C. E. Hoyle, T. Y. Lee and T. Roper, J. Polym. Sci. A Polym. Chem., 2004, 42, 5301-5338.

6. A. B. Lowe, Polym. Chem., 2010, 1, 17-36.

7. B. D. Fairbanks, T. F. Scott, C. J. Kloxin, K. S. Anseth and C. N. Bowman, Macromolecules, 2009, 42, 211-217.

8. R. Hoogenboom, Angew. Chem. Int. Ed., 2010, 49, 34153417.

9. B. Roberts, Chem. Soc. Rev., 1999, 28, 25-35.

$10 . \quad$ R. P. Allen, B. P. Roberts and C. R. Willis, J. Chem. Soc., Chem. Commun., 1989, 1387-1388.

11. S. J. Cole, J. N. Kirwan, B. P. Roberts and C. R. Willis, J. Chem. Soc., Perkin Trans. 1, 1991, 103-112.
12. H.-S. Dang and B. P. Roberts, Tetrahedron Lett., 1999, 40, 4271-4274.

13. H.-S. Dang, P. Franchi and B. P. Roberts, Chem. Commun., 2000, 499-500.

14. M. Uchiyama, K. Satoh and M. Kamigaito, Chem. Commun. 2019, 55, 5327-5330.

15. D. N. Amato, D. V. Amato, Y. Adewunmi, O. V. Mavrodi, K. H. Parsons, S. N. Swilley, D. A. Braasch, W. D. Walker, D. V. Mavrodi and D. L. Patton, ACS Applied Bio Materials, 2018, 1, 1983-1991.

16. D. N. Amato, D. V. Amato, O. V. Mavrodi, W. B. Martin, S. N. Swilley, K. H. Parsons, D. V. Mavrodi and D. L. Patton, ACS Macro Letters, 2017, 6, 171-175.

17. D. V. Amato, D. N. Amato, L. T. Blancett, O. V. Mavrodi, W. B. Martin, S. N. Swilley, M. J. Sandoz, G. Shearer, D. V. Mavrodi and D. L. Patton, Acta Biomaterialia, 2018, 67, 196-205.

18. B. P. Roberts and T. M. Smits, Tetrahedron Lett., 2001, 42, 137-140.

19. B. P. Roberts and T. M. Smits, Tetrahedron Lett., 2001, 42, 3663-3666.

20. Y. Cai, H.-S. Dang and B. P. Roberts, J. Chem. Soc. Perkin Trans. I, 2002, 2449-2458.

21. H.-S. Dang, B. P. Roberts and D. A. Tocher, Org. Biomol. Chem., 2003, 1, 4073-4084.

22. A. J. Fielding, P. Franchi, B. P. Roberts and T. M. Smits, J. Chem. Soc., Perkin Trans. 2, 2002, 155-163.

23. S. Chatterjee and S. Ramakrishnan, Chem. Commun., 2013, 49, 11041-11043.

24. N. B. Cramer, S. K. Reddy, A. K. O'Brien and C. N. Bowman, Macromolecules, 2003, 36, 7964-7969.

$25 . \quad$ S. K. Reddy, N. B. Cramer and C. N. Bowman, Macromolecules, 2006, 39, 3681-3687.

26. R. Batchelor, T. Messer, M. Hippler, M. Wegener, C. Barner-Kowollik and E. Blasco, Adv. Mater., 2019, 31, 1904085.

27. A. Romano, A. Angelini, E. Rossegger, G. Palmara, M. Castellino, F. Frascella, A. Chiappone, A. Chiadò, M. Sangermano, S. Schlögl and I. Roppolo, Macromol. Rapid Commun., 2020, 41, 2000084.

28. B. Steyrer, P. Neubauer, R. Liska and J. Stampfl, Materials (Basel), 2017, 10, 1445. 